\title{
Mechanical dyssynchrony in acute heart failure: A marker and a target?
}

\author{
G. Titus Ngeno, MD, ${ }^{a}$ Salvador Borges-Neto, $M D,{ }^{a, b, d}$ and Marat Fudim, $M D^{a, c}$ \\ a Duke Department of Medicine and Division of Cardiology, Durham, NC \\ b Duke Department of Radiology and Division of Nuclear Medicine, Durham, NC \\ c Duke Clinical Research Institute, Durham, NC \\ d Duke Heart Center, Durham, NC
}

Received Nov 30, 2020; accepted Nov 30, 2020

doi: $10.1007 / \mathrm{s} 12350-020-02468-8$

\begin{tabular}{|c|c|c|c|}
\hline$\overline{\text { Abb }}$ & tions & MPI & Myocardial perfusion imaging \\
\hline $\mathrm{BW}$ & Phase bandwidth & & \\
\hline $\mathrm{HF}$ & Heart failure & & \\
\hline
\end{tabular}

\section{See related article, pp. 140-149}

Ventricular dyssynchrony is the state of differential contraction and relaxation between the walls of the ventricle, leading to impairment in contractile efficiency. ${ }^{1}$ Interventricular dyssynchrony occurs when the timing of contraction between the right and left ventricles is mismatched, while intraventricular dyssynchrony refers to alterations in temporal contractility among regions of the left ventricle wall. Ventricular dyssynchrony is attributed to changes in both electrical signal conduction and inherent myocardial pathology such as scar, causing a mechanical impairment in contractility. Although both electrical dyssynchrony and mechanical dyssynchrony often occur together, both can also occur separately. ${ }^{2}$

Left ventricular electrical dyssynchrony (LVED) characterized by a QRS duration $>120 \mathrm{~ms}$ has been associated with an increased risk of major adverse cardiac events among patients with chronic heart failure. ${ }^{3}$ Furthermore, among chronic heart failure patients with LVED, improvement in cardiac function and symptoms after cardiac resynchronization therapy (CRT) has been

Reprint requests: Salvador Borges-Neto, MD, Duke Department of Radiology and Division of Nuclear Medicine, 2301 Erwin Road, Durham, NC 27710; Salvador.borgesneto@dm.duke.edu J Nucl Cardiol 2021;28:150-2.

$1071-3581 / \$ 34.00$

Copyright (C) 2021 American Society of Nuclear Cardiology. shown and incorporated into treatment guidelines. ${ }^{4}$ This notwithstanding, approximately one-third of patients who receive CRT show no improvement, highlighting the heterogeneity in causes of ventricular dyssynchrony beyond conduction delays. ${ }^{2}$

Left ventricular mechanical dyssynchrony (LVMD) assessment using SEPCT enables characterization of intraventricular mechanical dyssynchrony based on variations in regional wall thickness over the cardiac cycle independent of LVED. $^{5}$ Change in regional myocardial thickness as measured by photon count density varies with the cardiac cycle phase. Re-construction of volumetric frames enables temporal assessment of phase changes. Measures of phase standard deviations (PSD) and phase band width (PBW) distributions are reproducible and wider deviations are associated with poorer outcomes. ${ }^{6-8}$ The prognostic value of LVMD among patients with chronic heart failure as well as comorbidities such as coronary artery disease and diabetes has been highlighted. ${ }^{9,10}$

Among patients with acute heart failure, maladaptive physiologic changes in ventricular pre-load and afterload can trigger LVED and LVMD by accentuation of underlying pathology such as ischemia or by unmasking of pre-existing dyssynchrony. ${ }^{11}$ LVED is associated with severity of LVMD and an increased risk of ventricular arrhythmia. ${ }^{12}$ LVED has a linear correlation with severity of ventricular dysfunction, while greater LVMD is associated with a more reversible hemodynamic profile among patients with cardiogenic shock. ${ }^{13}$ Notably, even in acute presentations, LVED and severity of LVMD do not always correlate. LVMD 
often precedes onset of LVED or clinical symptoms of acute decompensated heart failure. ${ }^{9}$ Among patients with acute heart failure, LVMD is a dynamic process and a greater degree of LVMD is associated with a more reversible hemodynamic profile. ${ }^{11}$

In this issue, Zhou et al explore the prognostic value of integrative analysis of LVED and LVMD among patients with acute heart failure. The study demonstrates that both LVED as reflected by QRS duration $>120 \mathrm{~ms}$ and LVMD as reflected by $\mathrm{PBW}>77.76^{\circ}$ independently predict outcomes over an average period of 2.3 years. They also observed a higher accuracy in adverse outcome prediction by combining both measures. ${ }^{14}$ The integrated analysis, and subsequent superior predictive value, reinforces the growing understanding that there are several pathologic mechanisms underlying ventricular dyssynchrony. ${ }^{15}$ The novelty of this study lies in the acuity of the patients prospectively enrolled into the study. In contrast, prior studies evaluated LVED and LVMD in the context of chronic heart failure.

An important finding to consider is the study by Zhou et al, where it is observed that LVEF, widely recognized a predictive of heart failure outcomes, was not significantly associated with clinical events. This finding may be reflective of the small study sample size and clinical definition of LV dysfunction at enrollment into the study. Also noteworthy is the heterogeneity in heart failure phenotypes among patients enrolled in this study. Though the study adjusts for several factors that influence response to therapy and longitudinal outcomes among patients with heart failure, the findings are not broadly generalizable. Consequently, the prognostic value of integrative analysis of LVED and LVMD should be viewed through this lens as highlighted by the authors in the study limitations.

Integrative analysis of LVED and LVMD sheds light on future opportunities to understand the implications of hemodynamic reserve and reversibility of ventricular dyssynchrony. There are also opportunities to apply integrative analysis towards discerning prognostic differences based on heart failure sub-types. The potential role of diastolic dyssynchrony in heart failure for example remains to be elucidated. ${ }^{10}$ Importantly, by building on the body of knowledge highlighted in other studies on ventricular dyssynchrony, integrative analysis opens a new window for identification of patients who are likely to benefit from improved patient selection for CRT. ${ }^{16}$ There is a need for larger multicenter randomized studies in order to build generalizable prognostication models. In addition to predicting adverse outcomes, it is essential that future studies shed light on the pathogenesis of mechanical dyssynchrony and the implications on medical therapy. Ongoing experience with cardiac resynchronization therapies for chronic heart failure is teaching us that improving electrical dyssynchrony without a parallel improvement in mechanical dyssynchrony has a blunted impact on outcomes. Whether these lessons can be translated to acute heart failure and mechanical dyssynchrony can establish itself not only as a prognosticator but also as a treatment target remains to be determined.

\section{Disclosures}

Fudim is supported by a Mario Family Award, Duke Chair's Award, Bayer, Translating Duke Health Award; he receives consulting fees from AstraZeneca, AxonTherapies, CVRx, Daxor, Edwards LifeSciences, Galvani, NXT Biomedical, Respicardia.

\section{References}

1. Fudim M, Dalgaard F, Fathallah M, et al. Mechanical dyssynchrony: How do we measure it, what it means, and what we can do about it. J Nucl Cardiol 2019. https://doi.org/10.1007/s12350-01901758-0.

2. Fudim M, Borges-Neto S. A troubled marriage: When electrical and mechanical dyssynchrony don't go along. J Nucl Cardiol Off Publ Am Soc Nucl Cardiol 2019;26:1240-2. https://doi.org/10.10 07/s12350-018-1227-6.

3. Cheng A, Helm RH, Abraham TP. Pathophysiological mechanisms underlying ventricular dyssynchrony. Europace 2009;11:v10-4. https://doi.org/10.1093/europace/eup272.

4. Russo Andrea M, Stainback Raymond F, Bailey Steven R, et al. ACCF/HRS/AHA/ASE/HFSA/SCAI/SCCT/SCMR 2013 appropriate use criteria for implantable cardioverter-defibrillators and cardiac resynchronization therapy. J Am Coll Cardiol 2013;61:1318-68. https://doi.org/10.1016/j.jacc.2012.12.017.

5. Zafrir N. Left ventricular mechanical dyssynchrony graduation of myocardial perfusion gated SPECT phase analysis: What next. J Nucl Cardiol 2018;25:1009-11. https://doi.org/10.1007/s12350-01 6-0717-7.

6. Bader H, Garrigue S, Lafitte $S$, et al. Intra-left ventricular electromechanical asynchrony. A new independent predictor of severe cardiac events in heart failure patients. J Am Coll Cardiol 2004;43:248-56. https://doi.org/10.1016/j.jacc.2003.08.038.

7. Malhotra S. Dyssynchrony as a marker of adverse prognosis among patients with coronary artery disease and heart failure. $\mathbf{J}$ Nucl Cardiol 2020;27:1633-6. https://doi.org/10.1007/s12350-019 -01945-Z.

8. Rochlani Y, Khan MH, Gerard P, Jain D. Left ventricular dyssynchrony in diabetes mellitus. J Nucl Cardiol 2020;27:164951. https://doi.org/10.1007/s12350-018-01519-5.

9. Modin D, Biering-Sørensen SR, Møgelvang R, et al. Prognostic importance of left ventricular mechanical dyssynchrony in predicting cardiovascular death in the general population. Circ Cardiovasc Imaging 2018;11:e007528. https://doi.org/10.1161/CI RCIMAGING.117.007528.

10. Fudim M, Fathallah M, Shaw LK, et al. The prognostic value of diastolic and systolic mechanical left ventricular dyssynchrony among patients with coronary artery disease and heart failure. $\mathrm{J}$ Nucl Cardiol Off Publ Am Soc Nucl Cardiol 2020;27:1622-32. h ttps://doi.org/10.1007/s12350-019-01843-4.

11. Mullens W, Borowski AG, Curtin R, et al. Mechanical dyssynchrony in advanced decompensated heart failure: Relation to hemodynamic responses to intensive medical therapy. Heart 
Rhythm 2008;5:1105-10. https://doi.org/10.1016/j.hrthm.2008.04. 004.

12. Park S-J, On YK, Byeon K, et al. Short- and long-term outcomes depending on electrical dyssynchrony markers in patients presenting with acute heart failure: Clinical implication of the firstdegree atrioventricular block and QRS prolongation from the Korean Heart Failure Registry. Am Heart J 2013;165:57-64.e2. h ttps://doi.org/10.1016/j.ahj.2012.10.009.

13. Tanaka H, Tanabe M, Simon MA, et al. Left ventricular mechanical dyssynchrony in acute onset cardiomyopathy: Association of its resolution with improvements in ventricular function. JACC Cardiovasc Imaging 2011;4:445-56. https://doi.org/10.101 6/j.jcmg.2011.02.012.

14. Zhou Y, He Z, Liao S, et al. Prognostic value of integrative analysis of electrical and mechanical dyssynchrony in patients with acute heart failure. J Nucl Cardiol Off Publ Am Soc Nucl Cardiol 2020. https://doi.org/10.1007/s12350-020-02429-1.

15. Prem Soman. Mechanistic insights from prognostic studies of left ventricular dyssynchrony. Circ Cardiovasc Imaging 2018;11:e008186. https://doi.org/10.1161/CIRCIMAGING.118. 008186.

16. Fudim M, Borges-Neto S. Moving towards a synchronized left ventricle. J Nucl Cardiol Off Publ Am Soc Nucl Cardiol 2020;27:431-3. https://doi.org/10.1007/s12350-019-01749-1.

Publisher's Note Springer Nature remains neutral with regard to jurisdictional claims in published maps and institutional affiliations. 